\title{
Mixed ion-electron transport in organic electrochemical transistors $\odot$
}

Cite as: Appl. Phys. Lett. 117, 080501 (2020); https://doi.org/10.1063/5.0012599

Submitted: 03 May 2020 . Accepted: 03 August 2020. Published Online: 24 August 2020

Deyu Tu (D), and Simone Fabiano (D)

\section{COLLECTIONS}

F This paper was selected as Featured

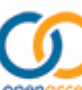

\section{ARTICLES YOU MAY BE INTERESTED IN}

Effect of channel thickness on noise in organic electrochemical transistors

Applied Physics Letters 117, 073302 (2020); https://doi.org/10.1063/5.0019693

Perspective on the pressure-driven evolution of the lattice and electronic structure in perovskite and double perovskite

Applied Physics Letters 117, 080502 (2020); https://doi.org/10.1063/5.0014947

A Perspective on interfacial engineering of lithium metal anodes and beyond

Applied Physics Letters 117, 080504 (2020); https://doi.org/10.1063/5.0018417

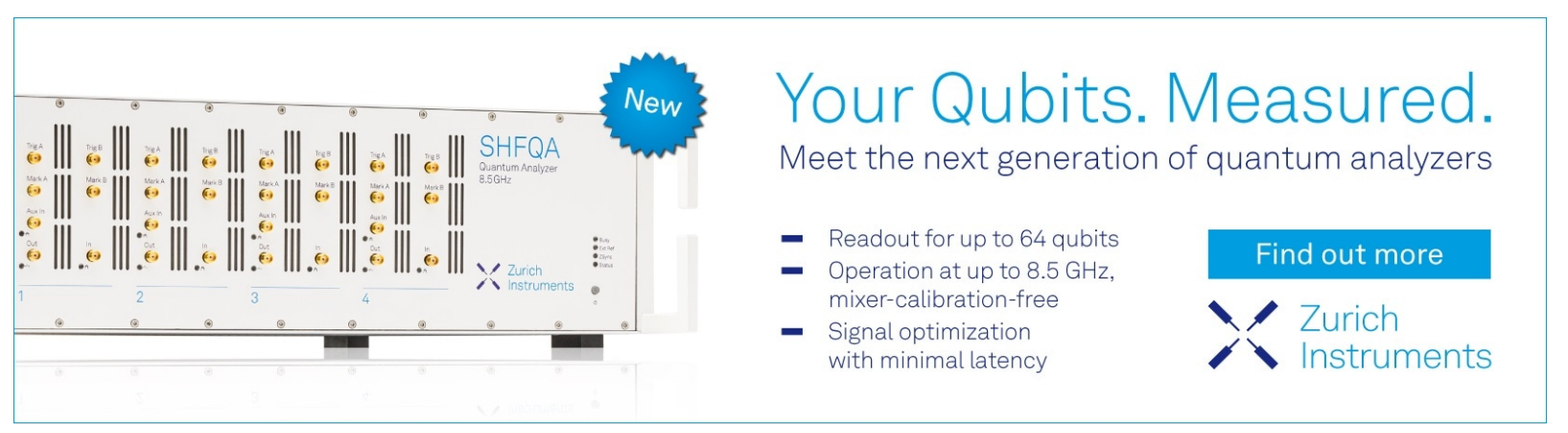




\title{
Mixed ion-electron transport in organic electrochemical transistors
}

\author{
Cite as: Appl. Phys. Lett. 117, 080501 (2020); doi: 10.1063/5.0012599 \\ Submitted: 3 May 2020 - Accepted: 3 August 2020 . \\ Published Online: 24 August 2020
}

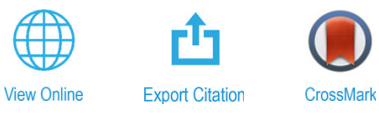

\section{${\text { Deyu } \mathrm{Tu}^{\mathrm{a})} \text { (D) and Simone Fabiano }}^{\text {b) }}$ (D)}

\author{
AFFILIATIONS \\ Laboratory of Organic Electronics, Department of Science and Technology, Linköping University, SE-601 74 Norrköping, Sweden

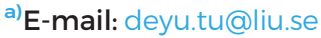 \\ ${ }^{\text {b) }}$ Author to whom correspondence should be addressed: simone.fabiano@liu.se
}

\begin{abstract}
Organic electrochemical transistors (OECTs) have shown great promise in a variety of applications ranging from digital logic circuits to biosensors and artificial synapses for neuromorphic computing. The working mechanism of OECTs relies on the mixed transport of ionic and electronic charge carriers, extending throughout the bulk of the organic channel. This attribute renders OECTs fundamentally different from conventional field effect transistors and endows them with unique features, including large gate-to-channel capacitance, low operating voltage, and high transconductance. Owing to the complexity of the mixed ion-electron coupling and transport processes, the OECT device physics is sophisticated and yet to be fully unraveled. Here, we give an account of the one- and two-dimensional drift-diffusion models that have been developed to describe the mixed transport of ions and electrons by finite-element methods and identify key device parameters to be tuned for the next developments in the field.
\end{abstract}

(C) 2020 Author(s). All article content, except where otherwise noted, is licensed under a Creative Commons Attribution (CC BY) license (http:// creativecommons.org/licenses/by/4.0/). https://doi.org/10.1063/5.0012599

Organic electrochemical transistors (OECTs) have been the subject of intensive research in the past five years, largely propelled by the continuous blossoming of novel applications. ${ }^{1-3}$ To date, OECTs have proven their value as chemical and biological sensors, ${ }^{4-10}$ amplifiers for cell culture analysis ${ }^{6,11}$ and medical diagnostics, ${ }^{12}$ printable digital logic circuits for the Internet of Things, ${ }^{13-16}$ or non-volatile memories for neuromorphic computing, ${ }^{17-19}$ to name just a few. This speedy move toward real-world applications has been enabled by a multidisciplinary research effort to better understand the materials, processing, and device/architecture requirements needed to achieve high levels of transistor performance.

In OECTs, the electroactive channel material bridging the source and drain electrodes is in direct contact with an electrolyte (Fig. 1). The soft and permeable nature of the organic semiconductor (OS) layer enables ions in the electrolyte to penetrate the bulk of the transistor channel upon application of an electric field. This leads to an electrochemical accumulation (or depletion) of charges in the semiconducting material and, thus, to a modulation of the transistor channel conductance. Hence, OECTs own their functioning to a reversible ion exchange and charge compensation process, which occurs throughout the organic semiconductor layer. ${ }^{20}$ This attribute makes the OECTs fundamentally different from the traditional organic field-effect transistors (OFETs), where modulation of the channel conductance is achieved by an electrostatic buildup of charges at the semiconductor and gate dielectric interface. ${ }^{21}$ The resulting large gateto-channel capacitance endows the OECTs with two unique features: (i) low operating voltage $<1 \mathrm{~V}$ (Ref. 22) and (ii) high transconductance. ${ }^{23}$ It appears, therefore, evident that an efficient ion and electron transport is of paramount importance for the functioning of OECTs.

Among the numerous mixed ion-electron conducting polymers that have been developed and reported to date as efficient channel materials for OECTs, ${ }^{3,24-26}$ PEDOT:PSS [viz., poly(3,4-ethylenedioxythiophene) doped with poly(styrene sulfonate)] is the most notable example of electroactive polymers, which yields high transistor performance. $^{24}$ The volumetric doping-dedoping of PEDOT:PSS films results in a modulation of the electrical conductivity by several orders of magnitude with consequently high transconductance. As PEDOT:PSS is highly conducting in its pristine state, the resulting OECTs operate in the depletion mode. In contrast, accumulation mode OECTs utilize mixed ion-electron conductors that are undoped in their pristine state as the channel material. The sophisticated ionic and electronic interactions and coupled transport properties in these mixed ion-electron conductors and corresponding OECTs require a special understanding that goes beyond knowledge derived for either electronic or ionic processes only. While several semi-empirical models have been proposed to describe the behaviors of OECTs, ${ }^{27}$ here we 


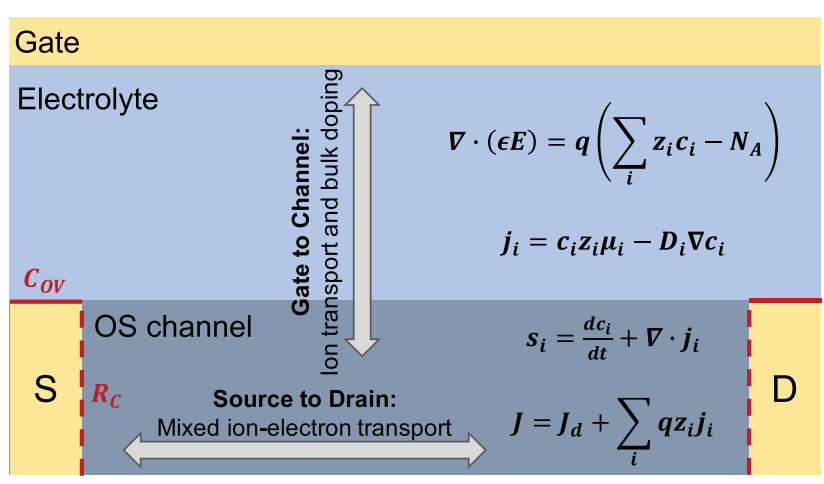

FIG. 1. In OECTs, ions inside the electrolyte are pushed toward the channel by a gate voltage bias, with a consequent doping (dedoping) and accumulation (depletion) of charges in the OS channel material. lons and electrons are then transported along the channel under the influence of the source-drain electrical field. A set of drift-diffusion equations describing the transport of ionic and electronic charge carriers is reported for both the electrolyte and the OS channel. Underexplored overlap capacitance $\left(\mathrm{C}_{\mathrm{OV}}\right)$ and contact resistance $\left(R_{\mathrm{C}}\right)$ effects are highlighted in red.

focus on multi-dimensional drift-diffusion models, as a tool to explore mixed ion-electron transport in OECTs by means of finite-element methods and to understand the interrelation between material parameters and device performance.

In this Perspective, we delve into the mechanisms of mixed ionelectron transport in OECTs. We provide an overview of the existing models describing ion-electron coupling and transport in the vertical (gate to channel) and horizontal (drain to source) directions and describe how these models, in particular, the drift-diffusion models, have advanced our understanding of the OECT working principle. Finally, we identify the incipient or even unexplored device parameters (contact resistance and parasitic capacitance) that are to be addressed for the next developments in this field.

Electrolytes are ion-conducting media, which play a key role in the transport of ions from the gate to the channel. Under a gate-tochannel bias, ion transport in the electrolyte is generally interpreted in terms of the drift-diffusion process, ${ }^{28}$ although this greatly depends on the materials. In liquid electrolytes, ions move together with the associated solvation shell, while in solvent-free polymer electrolytes, ions move through coordination sites assisted by the local segmental motion of the host polymer chain. ${ }^{28}$ Due to the large variety of electrolytes typically used in OECTs, the dependence of ion transport on materials properties and manufacturing processes is yet to be fully understood and it is typically modeled through inevitable simplifications. In one of the first attempts, Bernards and Malliaras (here referred to as the B-M model) proposed an equivalent ionic circuit for the gate-to-channel ionic coupling, in which only one resistor $\left(R_{\mathrm{S}}\right)$ is used to model the transport of ions in the electrolyte. ${ }^{29}$ Since then, the use of equivalent circuits has been a common approach to model the gate-to-channel interface in OECTs, and it has proven useful to analyze the device transient behaviors. For instance, Faria et al. expanded the B-M's ionic circuit by introducing a charge transfer resistance $\left(R_{\mathrm{CT}}\right)$ in parallel to the channel capacitance $\left(C_{\mathrm{CH}}\right) \cdot{ }^{30}$ By adding an extra circuit loop consisting of a resistor $\left(R_{\mathrm{bl}}\right)$ and a capacitor $\left(C_{\mathrm{bl}}\right)$ in parallel, Faria's model could also simulate the transient behavior of OECTs in which the channel is functionalized with partially permeable membranes. Gentile et al. replaced the charge transfer resistance $R_{\mathrm{CT}}$ with a Warburg impedance element $\left(Z_{\mathrm{W}}\right)$, which is often used in electrochemical impedance spectroscopy to account for the diffusion response of the ionic species. ${ }^{31}$ Another empirical impedance, a constant phase element $\left(C_{\mathrm{CPE}}\right)$, is also typically used in the equivalent circuits to account for ion migration at the electrolyte-organic semiconductor interface. ${ }^{32}$ Lago et al. developed an equivalent circuit in which every circuit element represents a particular physical layer or interface in OECTs. ${ }^{33}$ For example, they used a Warburg impedance element $Z_{\mathrm{W}}$ to account for the diffusion of ions across the electrolyte and a de Levie impedance to explain the percolation of ionic species. Finally, Andersson Ersman et al. used two resistors, one capacitor, and one diode in a SPICE model for OECTs to describe the transport of ions at the electrolyte-polymer interface and to reproduce the resulting OECT electrical characteristics. ${ }^{14}$ One resistor $\left(R_{1}\right)$ represents the drift of ions under gate bias, while the second resistor $\left(R_{2}\right)$ and the capacitor $\left(C_{1}\right)$ represent the diffusion of ions when the gate is grounded. The diode $\left(D_{1}\right)$ is activated when $C_{1}$ discharges through both $R_{1}$ and $R_{2}$. The model was then able to reproduce the asymmetric and slow switching behaviors typically observed in the experiments. All the equivalent circuit models discussed above have proven useful to describe the experimentally measured OECT transient response, and further adjustments can be made to deal with specific device/material modifications (e.g., gate/channel functionalization ${ }^{30}$ ) although a deeper understanding of the relationship between device behaviors and materials properties is still lacking.

To quantitatively understand ion transport in electrolytes, one can define an ionic conductivity $\sigma_{\text {ionic }}$, which takes into account the contribution from each mobile ionic species $\left(\sigma_{i}\right)$ as follows: ${ }^{34}$

$$
\sigma_{\text {ionic }}=\sum_{i} \sigma_{i}=\sum_{i} q\left|z_{i}\right| n_{i} \mu_{i}
$$

where $q$ is the elementary charge, $z_{i}$ is the ion valence, $n_{i}$ is the ion density, and $\mu_{i}$ is the ion mobility. The latter is related to the ion diffusivity $D_{i}$ by the Einstein relation ${ }^{34}$

$$
D_{i}=\mu_{i} \frac{k_{B} T}{q},
$$

where $k_{\mathrm{B}}$ is the Boltzmann constant and $T$ is the temperature.

Using the ion diffusivity $D$, Coppedé et al. developed a model where the diffusion of ions in the electrolyte is considered to follow the drift-diffusion equation, ${ }^{35}$

$$
J=-D\left(\nabla \mathrm{c}+\frac{z F}{R T} c \nabla \varphi\right),
$$

where $J$ is the diffusion flux (ionic current) density, $c$ is the ion concentration, $z$ is the ion valence, $F$ is the Faraday constant, $R$ is the universal gas constant, and $\varphi$ is the electric potential. The model is used to distinguish various ions from a complex mixture, on the basis of their diffusivities. By fitting the experimentally measured OECT transient curves, the physical characteristics of the ionic species transported in the electrolyte can be determined.

Tybrandt et al. developed a 1D drift-diffusion model for the electrolyte/conducting polymer system. ${ }^{36}$ The complete model for ion transport is based on the Nernst-Planck drift-diffusion equation [Eq. 4(a)] describing the ionic species in the electrolyte phase, the continuity equation [Eq. 4(b)] governing the changes in concentrations, and 
the Poisson equation [Eq. 4(c)] describing the transport of ions, as follows:

$$
\begin{gathered}
j_{c_{ \pm}}=-D_{c_{ \pm}}\left(\nabla c_{ \pm} \pm f c_{ \pm} \nabla \varphi_{c_{ \pm}}\right), \\
\nabla j_{c_{ \pm}}=-\frac{d c_{ \pm}}{d t}, \\
-\frac{\varepsilon}{F} \nabla^{2} \varphi_{c}=c_{+}-c_{-},
\end{gathered}
$$

where $f=z F / R T$ as in Eq. (3), $c_{ \pm}$denotes the cation or anion concentration, and $t$ is the time. Together with another set of equations for the conducting polymer phase (see discussion below), the model provides a coherent theoretical framework to understand the physical connections between the OECT behaviors and the materials parameters, such as the ion diffusivity, energy levels, and ion/charge carrier density/concentrations.

Bulk doping or volumetric charging of the (semi)conducting polymer phase in the channel is often modeled as a potentialdependent capacitance in the equivalent-circuit models discussed above. While this charging process was traditionally thought of as being purely redox (Faradaic process), ${ }^{2}$ e.g.,

$$
\text { PEDOT }^{+}: \text {PSS }^{-}+\mathrm{M}^{+} \stackrel{e^{-}}{\longleftrightarrow} \text { PEDOT }^{0}+\mathrm{M}^{+}: \text {PSS }^{-},
$$

where PEDOT:PSS is the conducting polymer blend and $\mathrm{M}^{+}$are the cations (e.g., $\mathrm{Na}^{+}$), recent works have suggested that coupled electron-ion motion and double layer charging (capacitive process) are instead predominant in conducting polymers. ${ }^{37}$ Hence, the charging of mixed ion-electron conducting polymers is often referred to as pseudocapacitive. Nevertheless, because of the complexity of the ionicelectronic coupling process, an accurate description of the charging phenomena is difficult and it requires additional experimental and theoretical work to be done.

Due to the similarity with the conventional field effect transistors (FETs), the electrical characteristics of OECTs are often modeled by means of equivalent circuits, which consist of a FET and other passive components. Friedlein et al., ${ }^{38}$ for example, used a p-type junction fieldeffect transistor (JFET) instead of an OECT in the equivalent circuit, while Andersson Ersman et al. developed a model ${ }^{14}$ that comprises a $p$ type depletion-mode metal-oxide-semiconductor FET (MOSFET). Both steady-state and transient behaviors of PEDOT:PSS-based OECTs could be simulated by means of these models, despite being the working mechanism of OECTs very different from that of JFETs or MOSFETs.

Charge transport in organic semiconductors has been extensively investigated. While single-crystal semiconductors ${ }^{39}$ or conjugated polymers with torsion-free backbone conformation ${ }^{40}$ have shown classic band-like transport, amorphous or highly disordered semiconductors are mainly characterized by a variable range hopping transport mechanism where charges hop among localized states. ${ }^{41}$ The energy level in disordered thin films of organic semiconductors is usually described as a non-uniform density of states (DOS), which is either exponential or Gaussian. Together with the Fermi function $f\left(E, E_{F}\right)$, the charge carrier density $p$ is written as ${ }^{42,43}$

$$
p=\int \operatorname{DOS}(E) f\left(E, E_{F}\right) d E,
$$

where $\operatorname{DOS}(E)=\frac{N_{t}}{\sqrt{2 \pi} \sigma_{G}} \exp \left(-\frac{E^{2}}{2 \sigma_{G}^{2}}\right)$ for a Gaussian DOS, ${ }^{42}$ or $\operatorname{DOS}(E)=\frac{N_{t}}{k_{B} T_{0}} \exp \left(\frac{E}{k_{B} T_{0}}\right) \quad$ for $\quad$ an exponential $\quad$ DOS, ${ }^{43}$
$f\left(E, E_{F}\right)=\frac{1}{e^{\left(E-E_{F}\right) / k_{B} T}+1}, E$ is the energy of localized states, $E_{F}$ is the Fermi level, $N_{t}$ is the total number of localized states per unit volume, $\sigma_{\mathrm{G}}$ is the width of the Gaussian DOS, and $T_{0}$ indicates the width of the distribution function.

The non-uniform DOS typically results in a charge carrier mobility that is dependent on voltage and temperature, and it can be simplified as ${ }^{44}$

$$
\mu=\mu_{0}\left(V_{G}-V_{T}\right)^{\gamma}
$$

where the exponent $\gamma=2\left(\frac{T_{0}}{T}-1\right), \mu_{0}$ is the mobility at a low electric field, $V_{\mathrm{G}}$ is the gate voltage, and $V_{\mathrm{T}}$ is the threshold voltage (accumulation mode).

The current density $J_{x}$, originating from the drift of charges, is given by the charge carrier mobility $\mu$, the charge density $p(x)$, and the electrical field $\partial V(x) / \partial x$ at the position $x$ in the transistor channel ${ }^{29}$

$$
J_{x}=\frac{\mu q p(x)(\partial V(x))}{\partial x} .
$$

For OECTs operated in the depletion mode, such as, for example, PEDOT-based OECTs, the effective charge density in a volume $v$ is ${ }^{29}$

$$
p=p_{0}\left(1-\frac{Q}{q p_{0} v}\right)
$$

where $Q$ is the total charge injected from the electrolyte and $p_{0}$ is the initial charge density. By integrating the current density equation along the channel, Bernards and Malliaras obtained the steady-state drain current $I_{D}$ as follows: ${ }^{29}$

$$
\begin{gathered}
I_{D}=G\left[V_{D}-\frac{V_{G}^{2}}{2 V_{P}}\right] \text { for } V_{D}>V_{G}, \\
I_{D}=G\left[1-\frac{V_{G}-V_{D} / 2}{V_{P}}\right] V_{D} \text { for } V_{G}>V_{D}>V_{G}-V_{P}, \\
I_{D}=-G \frac{V_{D s a t}^{2}}{2 V_{P}} \text { for } V_{D}<V_{G}-V_{P},
\end{gathered}
$$

where $G$ is the conductance of the organic semiconductor thin films, $V_{\mathrm{D}}$ is the drain voltage, $V_{\mathrm{P}}$ is the pinch-off voltage (depletion mode), and $V_{\text {Dsat }}=V_{\mathrm{G}}-V_{\mathrm{P}}$. As the charge carrier mobility in the B-M model was voltage-independent, Friedlein et al. assumed an exponential DOS and introduced a voltage-dependent mobility. ${ }^{45}$

For OECTs operated in the accumulation mode, a drift model for charge carrier transport can be adopted from the akin electrolytegated organic field effect transistors (EGOFETs), ${ }^{46}$ by introducing a volumetric capacitance or a voltage-dependent electric double layer capacitance $\left(C_{\mathrm{EDL}}\right)$. For instance, Tu et al. extended the drift model to include the volumetric capacitance, yielding ${ }^{46}$

$$
\begin{aligned}
I_{D}= & \mu_{0} \frac{W d}{L}\left[C_{0} \frac{V_{G T}^{\gamma+2}-\left(V_{G T}-V_{D}\right)^{\gamma+2}}{\gamma+2}\right. \\
& +C_{i} \frac{\left.V_{G T}^{\gamma+\chi+2}-\left(V_{G T}-V_{D}\right)^{\gamma+\chi+2}\right]}{\gamma+\chi+2} \text { for } V_{D}<V_{G T}, \\
I_{D}= & \mu_{0} \frac{W d}{L}\left[C_{0} \frac{V_{G T}^{\gamma+2}}{\gamma+2}+C_{i} \frac{V_{G T}^{\gamma+\chi+2}}{\gamma+\chi+2}\right] \text { for } V_{D}>V_{G T},
\end{aligned}
$$

where $\chi$ is the $C_{\mathrm{EDL}}$ voltage-dependent factor, $C_{0}$ is the volumetric capacitance independent of voltage, $C_{\mathrm{i}}$ is the volumetric capacitance 
dependent on voltage, $W$ is the channel width, $L$ is the channel length, and $d$ is the channel thickness.

The drift models only consider the charge carriers (holes or electrons) in the channel, while in reality ions from the electrolyte penetrate into the OECT channel material and bulk dope it. The 1D model presented in Ref. 30 combines the charge (hole) transport and the ion transport in the conducting polymer phase (channel) by applying the drift-diffusion equations to charge carriers (holes and ions), as follows: ${ }^{36}$

$$
\begin{gathered}
j_{p}=-D_{p}\left(\nabla \mathrm{p} \pm f p \nabla\left(\varphi_{p}+\mu_{p} / q\right),\right. \\
\nabla j_{p}=-\frac{d p}{d t}, \\
-\frac{\varepsilon}{F} \nabla^{2} \varphi_{p}=p-\left(\varphi_{p}-\varphi_{c}\right) C_{V},
\end{gathered}
$$

where $C_{\mathrm{V}}$ is the volumetric capacitance, $p$ is the charge carrier density ( $p$ denotes holes for a $p$-type conductor), and $c$ is the ion density. In this model, both ions and holes in the channel are considered to describe a mixed ionic and electronic carrier transport. Prior to this model, Robinson et al. ${ }^{47}$ proposed a semi-empirical model based on the Nernst equation to describe the equilibrium relationship between doping and applied potential, to explain the steady-state current saturation in PEDOT:PSS-based OECTs,

$$
E-E_{0}=-\frac{R T}{z F} \ln \left(\frac{A_{\text {products }}}{A_{\text {reactants }}}\right),
$$

where $E$ is the applied potential, $E_{0}$ is the standard redox potential, and $A$ indicates the product of the activities of the reaction products or reactants. Similarly, Shirinskaya et al. modeled the steady-state charge distribution and electrical characteristics of PEDOT:PSS-based OECTs, by using a modified Nernst equation, which takes into account the total density of neutral PEDOT $p_{0}$, the density of oxidized $\mathrm{PEDOT}^{+} p_{\text {ox }}$, and the applied voltage with respect to the grounded source $V^{48}$

$$
p_{o x}=\frac{p_{0}}{1+\exp \left[\frac{q}{k T}\left(V-V_{R}\right)\right]},
$$

where $V_{\mathrm{R}}$ is the difference between the redox potential and the work function of PEDOT:PSS. Then, the drift-diffusion equation (aka Nernst-Planck equation) and the Poisson equation were used to model the moving "front" of the electrolyte/PEDOT:PSS system. The diffusivity of ions was omitted from this $1 \mathrm{D}$ model.

All the models discussed above are based on the use of equivalent circuits, classic drift models, or 1D numerical simulations. These models deal with different aspects of the OECT device physics, but they suffer from significant limitations. For instance, none of the models describes the steady-state, transient, and AC responses consistently, and none of them, with the exception of the SPICE model presented in Ref. 14, accounts for the asymmetric non-exponential pulse responses of OECTs due to asymmetry in the charging/discharging of the channel. More importantly, because of the physical constraints of the $1 \mathrm{D}$ models, ion movement is only considered in the direction perpendicular to the channel. However, movement of ions along the channel needs to be considered as well and this can only be done with 2D models. To overcome these limitations, Szymański et al. reported a 2D drift-diffusion model of OECTs by extending the finite-element modeling of semiconductor devices with ion transport. ${ }^{49}$ The driftdiffusion model consists of the Poisson equation [Eq. 15(a)] describing the transport of both ionic and electronic charge carriers, the current equation for each species [Eq. 15(b)] and the current density equation [Eq. 15(c)], which sum the contribution of both drift and diffusion, and the conservation (continuity) equation [Eq. 15(d)] describing charge conservation in the system,

$$
\begin{aligned}
\nabla \cdot(\epsilon E) & =q\left(\sum_{i} z_{i} c_{i}-N_{A}\right), \\
j_{i} & =c_{i} z_{i} \mu_{i}-D_{i} \nabla c_{i}, \\
J & =J_{d}+\sum_{i} q z_{i} j_{i}, \\
s_{i} & =\frac{d c_{i}}{d t}+\nabla \cdot j_{i},
\end{aligned}
$$

where $E=-\nabla \varnothing$ is the local electric field, $\varnothing$ is the local electrostatic potential, and $c_{i}$ is the local concentration, $z_{i}$ is the relative charge species, $N_{\mathrm{A}}$ is the local concentration of ionized acceptors, $J$ is the electric current density, $J_{d}=\epsilon(\partial E / \partial t)$ is the displacement current contribution, which is included in the transient and AC simulations, and $j_{i}$ is the flux of specie $i$. The source term $s_{i}$ can be used to model chemical reactions or charge carrier trapping. The diffusivity $D_{i}$ modified to include the steric potential is used to enforce maximum ion concentration $c_{i 0}$ in the system

$$
D_{i}=\mu_{i} \frac{k_{B} T}{q}\left(1-\frac{c_{i}}{c_{i o}}\right) .
$$

Meshing is a key step in finite-element modeling to ensure accuracy and convergence of the solutions within manageable computing power. Graded tensor-product meshes are used in the 2D model, and a minimum mesh spacing of $0.1 \mathrm{~nm}$ is typically used for electrical double layers. By fixing a set of physical parameters such as temperature, ion and hole mobilities, maximum ion and hole concentrations, ionized acceptor concentration, relative permittivity, and electrolyte concentration, the authors were able to model the steady-state, transient (asymmetric charging/discharging), and AC responses of PEDOT:PSS-based OECTs consistently with the experimental results. This $2 \mathrm{D}$ drift-diffusion model explains the OECT behaviors purely by the transport of charged species, including both ions and electrons (holes). It can also be further improved by considering (a) the asymmetry between cations and anions, (b) the different transport properties in the electrolyte and in the channel, (c) voltage or concentration dependent mobility/diffusivity, and (d) chemical reactions. The main challenge associated with this specific finite-element model is the realistic OECT dimensions. The electrical double layer thickness is $1 \mathrm{~nm}$, while the OECT channel length and width can be up to hundreds of micrometers. Hence, it becomes difficult to handle elements with extremely small volume and elements with very large aspect ratios when the model is built with the realistic dimensions of large-channel OECTs.

Recently, Kaphle et al. compared a 2D drift-diffusion model of depletion-mode OECTs with capacitive device models (equivalent-circuit and $1 \mathrm{D}$ models reported previously), ${ }^{50}$ suggesting that the ions follow an exponential distribution along the channel when a lateral ion movement is taken into account in the $2 \mathrm{D}$ model. The $2 \mathrm{D}$ driftdiffusion model simulates the gate-to-channel spatial configuration of 
hole concentration, cation concentration, potential, and carrier density and shows a large ion accumulation at the drain (for depletion-mode OECTs), which leads to an additional voltage drop. Although previous capacitive device models failed to predict this scenario, they can be complemented by setting a gate-capacitance exponentially dependent on potential or additional contact resistance at the drain to obtain similar results. ${ }^{50}$ However, the physical interpretation of the device mechanism is completely different. The findings suggest that $2 \mathrm{D}$ drift-diffusion models offer a better understanding of the spatial configuration of the various species (i.e., electrons, holes, ions) in OECTs and, thus, provide a clearer picture of the device operation mechanisms.

Forming Ohmic contacts between metal electrodes and organic (semi-)conductors has proven to be a challenge in order to further enhance the performance of organic transistors. ${ }^{51}$ All the contacts between metal and polymers are essentially Schottky with a barrier height that limits the charge injection, and thus it becomes one of the two major critical parameters (surprisingly, not the charge carrier mobility) limiting the switching speed/cutoff frequency of high performance organic FETs. ${ }^{52}$ Lowering the Schottky barrier height by engineering the metal-organic interface can produce fairly good Ohmic contacts, but it may also result in high leakage current for accumulation-mode transistors in the OFF-state. ${ }^{51}$ In the case of electrolyte-gated transistors, the electrochemical doping induced by ions penetrating into the semiconductor channel significantly contributes to reduce the device contact resistance, resulting in values that are up to two orders of magnitudes lower than those of traditional OFETs. ${ }^{53}$ This electrochemical doping process is highly dependent on the nature of the electrolyte, ${ }^{54}$ although the relationship between contact resistance and electrolyte is yet to be clearly understood. So far, there have been only three attempts to systematically investigate the role of contact resistance in OECTs, despite the significant impact this could have on the transistor performance. Kaphle et al. observed that contact resistance in PEDOT:PSS-based OECTs depends exponentially on the gate-to-source voltage, as in OFETs, and extended the B-M model to include the effect of contact resistance. ${ }^{55}$ Later, it was shown that the contact resistance contributes $20 \%$ of the total channel resistance for PEDOT:PSS depletion-mode OECTs, while it contributes only $2 \%$ in the case of $p$ type accumulation-mode OECTs. ${ }^{45}$ Recently, Paterson et al. investigated the effect of self-assembly monolayer (SAM) surface modification on the contact resistance in $n$-type accumulation-mode OECTs. ${ }^{56}$ They observed that contact resistance has a negative impact on the device performance and that SAM modification of source/drain contacts aids to lower the contact resistance by improving the surface electrode wettability and the semiconductor film quality. Thus, because of the limited number of studies, the role of contact resistance in OECTs and its dependence on material properties (i.e., electrolytes, channel conductors, and electrodes) and fabrication processes remain unclear.

In addition to contact resistance, the other important parameter limiting the switching speed of OECTs is the parasitic/overlap capacitance. The cutoff frequency (aka transit frequency, $f_{\mathrm{T}}$ ), which is the figure-of-merit defining how fast a transistor can be operated, is described as ${ }^{52}$

$$
f_{T}=\frac{g_{m}}{2 \pi\left(C_{G}+C_{o v}\right)},
$$

where $C_{\mathrm{G}}$ is the intrinsic gate-to-channel capacitance and $C_{\mathrm{ov}}$ is the parasitic contributions derived from the overlap of the gate electrode
TABLE I. Typical OECT figures of merit and relevant applications. The number of (C) qualitatively indicates the degree to which the applications depend on the various figures of merit.

\begin{tabular}{|c|c|c|c|}
\hline \multirow[b]{2}{*}{ Figure of merit } & \multicolumn{3}{|c|}{ Application } \\
\hline & Bioelectronics & Logics & Neuromorphics \\
\hline Transconductance (mS) & 0 & 0 & \\
\hline Cutoff frequency $(\mathrm{Hz})$ & 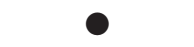 & 0 & 0 \\
\hline Operating voltage (V) & 0 & 0 & 0 \\
\hline State retention (s) & & & 0 \\
\hline Footprint $\left(\mu \mathrm{m}^{2}\right)$ & 0 & 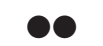 & 0 \\
\hline
\end{tabular}

with the source and drain contacts. The large bulk (volumetric) capacitance of electrolyte/conducting polymers is a double-edged sword enabling low voltage operation and high transconductance, but also inducing fairly low cutoff frequency. A natural approach to optimize the OECT performance is to increase the intrinsic gate-to-channel capacitance while suppressing the parasitic capacitance as much as possible. This quest for a large intrinsic gate capacitance has resulted in novel transistor architectures, such as the newly reported internalgated OECTs. ${ }^{57}$ Recently, Polyravas et al. investigated the impact of overlap capacitance in PEDOT:PSS-based OECTs and suggested that the cutoff frequency decreases by increasing the contact overlap, while the steady-state transconductance remains largely unaffected. ${ }^{58}$ These results indicate that minimal overlap is a key to obtain the best performing OECTs. To this end, the use of laser cutting could enable the fabrication of self-aligned OECTs with minimal contact overlap. ${ }^{59}$

Thanks to their unique features, including the ease of manufacturing, large gate-to-channel capacitance, low operating voltage, and high transconductance, OECTs offer an exciting opportunity for the development of future flexible large-area electronics. A list of applications is reported in Table I together with the associated figures of merit, which strongly depend on the OECT device physics presented in this Perspective. However, because of the complexity of the mixed ion-electron coupling and transport processes and their dependence on the conducting material properties, the OECT device physics is far from being clearly articulated. Here, we summarized the current effort (including ours), which has been devoted to understanding the OECT working principle, and proposed viable route (e.g., contact resistance and parasitic capacitance) to boost their performance (e.g., switching speed and long-term stability).

This work was financially supported by the Swedish Foundation for Strategic Research (No. SE13-0045), the Swedish Research Council (No. 2016-03979), ÅForsk (Nos. 18-313 and 19310), Olle Engkvists Stiftelse (No. 204-0256), and the Swedish Government Strategic Research Area in Materials Science on Functional Materials at Linköping University (Faculty Grant SFOMat-LiU No. 2009-00971).

\section{DATA AVAILABILITY}

Data sharing is not applicable to this article as no new data were created or analyzed in this study. 


\section{REFERENCES}

${ }^{1}$ H. S. White, G. P. Kittlesen, and M. S. Wrighton, J. Am. Chem. Soc. 106, 5375 (1984).

${ }^{2}$ D. Nilsson, M. Chen, T. Kugler, T. Remonen, M. Armgarth, and M. Berggren, Adv. Mater. 14, 51 (2002).

${ }^{3}$ H. Sun, M. Vagin, S. Wang, X. Crispin, R. Forchheimer, M. Berggren, and S, Fabiano, Adv. Mater. 30, 1704916 (2018).

${ }^{4}$ D. A. Bernards, G. G. Malliaras, G. E. S. Toombes, and S. M. Gruner, Appl. Phys. Lett. 89, 053505 (2006).

${ }^{5}$ C. Qian, L. Kong, J. Yang, Y. Gao, and J. Sun, Appl. Phys. Lett. 110, 083302 (2017).

${ }^{6}$ J. Rivnay, M. Ramuz, P. Leleux, A. Hama, M. Huerta, and R. M. Owens, Appl. Phys. Lett. 106, 043301 (2015).

${ }^{7}$ G. Scheiblin, R. Coppard, R. M. Owens, P. Mailley, and G. G. Malliaras, Adv. Mater. Technol. 2, 1600141 (2017).

${ }^{8}$ N. Y. Shim, D. A. Bernards, D. J. Macaya, J. A. DeFranco, M. Nikolou, R. M. Owens, and G. G. Malliaras, Sensors 9, 9896 (2009).

${ }^{9}$ P. O. Svensson, D. Nilsson, R. Forchheimer, and M. Berggren, Appl. Phys. Lett. 93, 203301 (2008).

${ }^{10}$ H. Tang, F. Yan, P. Lin, J. Xu, and H. L. W. Chan, Adv. Funct. Mater. 21, 2264 (2011).

${ }^{11}$ M. Braendlein, T. Lonjaret, P. Leleux, J.-M. Badier, and G. G. Malliaras, Adv. Sci, 4, 1600247 (2017).

${ }^{12}$ P. Leleux, J. Rivnay, T. Lonjaret, J. M. Badier, C. Bénar, T. Hervé, P. Chauvel, and G. G. Malliaras, Adv. Healthcare Mater. 4, 142 (2015).

${ }^{13}$ P. C. Hutter, T. Rothlander, G. Scheipl, and B. Stadlober, IEEE Trans. Electron Devices 62, 4231 (2015).

${ }^{14}$ P. Andersson Ersman, D. Westerberg, D. Tu, M. Nilsson, J. Åhlin, A. Eveborn, A. Lagerlöf, D. Nilsson, M. Sandberg, P. Norberg, M. Berggren, R. Forchheimer, and G. Gustafsson, Flexible Printed Electron. 2, 045008 (2017).

${ }^{15}$ P. A. Ersman, M. Zabihipour, D. Tu, R. Lassnig, J. Strandberg, J. Åhlin, M. Nilsson, D. Westerberg, G. Gustafsson, M. Berggren, R. Forchheimer, and S. Fabiano, Flexible Printed Electron. 5, 024001 (2020).

${ }^{16}$ P. Andersson Ersman, R. Lassnig, J. Strandberg, D. Tu, V. Keshmiri, R. Forchheimer, S. Fabiano, G. Gustafsson, and M. Berggren, Nat. Commun. 10, 5053 (2019)

${ }^{17}$ Y. Van De Burgt, E. Lubberman, E. J. Fuller, S. T. Keene, G. C. Faria, S. Agarwal, M. J. Marinella, A. Alec Talin, and A. Salleo, Nat. Mater. 16, 414 (2017).

${ }^{18}$ P. Gkoupidenis, D. A. Koutsouras, and G. G. Malliaras, Nat. Commun. 8, 15448 (2017).

${ }^{19}$ J. Y. Gerasimov, R. Gabrielsson, R. Forchheimer, E. Stavrinidou, D. T. Simon, M. Berggren, and S. Fabiano, Adv. Sci. 6, 1801339 (2019).

${ }^{20}$ J. Rivnay, S. Inal, A. Salleo, R. M. Owens, M. Berggren, and G. G. Malliaras, Nat. Rev. Mater. 3, 17086 (2018).

${ }^{21} \mathrm{H}$. Sirringhaus, Adv. Mater. 26, 1319 (2014).

${ }^{22}$ E. Zeglio, J. Eriksson, R. Gabrielsson, N. Solin, and O. Inganäs, Adv. Mater. 29, 1605787 (2017)

${ }^{23}$ D. Khodagholy, J. Rivnay, M. Sessolo, M. Gurfinkel, P. Leleux, L. H. Jimison, E. Stavrinidou, T. Herve, S. Sanaur, R. M. Owens, and G. G. Malliaras, Nat, Commun. 4, 2133 (2013).

${ }^{24}$ J. Rivnay, S. Inal, B. A. Collins, M. Sessolo, E. Stavrinidou, X. Strakosas, C. Tassone, D. M. Delongchamp, and G. G. Malliaras, Nat. Commun. 7, 11287 (2016).

${ }^{25}$ A. Giovannitti, C. B. Nielsen, D.-T. Sbircea, S. Inal, M. Donahue, M. R. Niazi, D. A. Hanifi, A. Amassian, G. G. Malliaras, J. Rivnay, and I. Mcculloch, Nat. Commun. 7, 13066 (2016).
${ }^{26}$ A. Giovannitti, D. T. Sbircea, S. Inal, C. B. Nielsen, E. Bandiello, D. A. Hanifi, M. Sessolo, G. G. Malliaras, I. McCulloch, and J. Rivnay, Proc. Natl. Acad. Sci. U. S. A. 113, 12017 (2016).

${ }^{27}$ J. T. Friedlein, R. R. McLeod, and J. Rivnay, Org. Electron. 63, 398 (2018).

${ }^{28}$ M. A. Ratner and D. F. Shriver, Chem. Rev. 88, 109 (1988).

${ }^{29}$ D. A. Bernards and G. G. Malliaras, Adv. Funct. Mater. 17, 3538 (2007).

${ }^{30}$ G. C. Faria, D. T. Duong, and A. Salleo, Org. Electron. 45, 215 (2017).

${ }^{31}$ F. Gentile, D. Delmonte, M. Solzi, M. Villani, S. Iannotta, A. Zappettini, and N. Coppedè, Org. Electron. 35, 59 (2016).

${ }^{32}$ O. Larsson, E. Said, M. Berggren, and X. Crispin, Adv. Funct. Mater. 19, 3334 (2009).

${ }^{33}$ N. Lago, A. Cester, N. Wrachien, M. Natali, S. D. Quiroga, S. Bonetti, M. Barbato, A. Rizzo, E. Benvenuti, V. Benfenati, M. Muccini, S. Toffanin, and G. Meneghesso, Org. Electron. 35, 176 (2016).

${ }^{34}$ B. D. Paulsen, K. Tybrandt, E. Stavrinidou, and J. Rivnay, Nat. Mater. 19, 13 (2020).

${ }^{35}$ N. Coppedè, M. Villani, and F. Gentile, Sci. Rep. 4, 4297 (2014).

${ }^{36}$ K. Tybrandt, I. V. Zozoulenko, and M. Berggren, Sci. Adv. 3, eaao3659 (2017).

${ }^{37} \mathrm{M}$. Berggren and G. G. Malliaras, Science 364, 233 (2019).

${ }^{38}$ J. T. Friedlein, M. J. Donahue, S. E. Shaheen, G. G. Malliaras, and R. R. McLeod, Adv. Mater. 28, 8398 (2016).

${ }^{39}$ N. A. Minder, S. Ono, Z. Chen, A. Facchetti, and A. F. Morpurgo, Adv. Mater. 24, 503 (2012).

${ }^{40}$ D. Venkateshvaran, M. Nikolka, A. Sadhanala, V. Lemaur, M. Zelazny, M. Kepa, M. Hurhangee, A. J. Kronemeijer, V. Pecunia, I. Nasrallah, I. Romanov, K. Broch, I. McCulloch, D. Emin, Y. Olivier, J. Cornil, D. Beljonne, and H. Sirringhaus, Nature 515, 384 (2014).

${ }^{41}$ N. Tessler, Y. Preezant, N. Rappaport, and Y. Roichman, Adv. Mater. 21, 2741 (2009).

${ }^{42}$ H. Bässler, Phys. Status Solidi B 175, 15 (1993).

${ }^{43}$ M. C. J. M. Vissenberg and M. Matters, Phys. Rev. B 57, 12964 (1998).

${ }^{44}$ O. Marinov, M. J. Deen, and R. Datars, J. Appl. Phys. 106, 064501 (2009).

${ }^{45}$ J. T. Friedlein, J. Rivnay, D. H. Dunlap, I. McCulloch, S. E. Shaheen, R. R. McLeod, and G. G. Malliaras, Appl. Phys. Lett. 111, 023301 (2017).

${ }^{46}$ D. Tu, L. Herlogsson, L. Kergoat, X. Crispin, M. Berggren, and R. Forchheimer, IEEE Trans. Electron Devices 58, 3574 (2011).

${ }^{47}$ N. D. Robinson, P. O. Svensson, D. Nilsson, and M. Berggren, J. Electrochem, Soc. 153, H39 (2006).

${ }^{48}$ A. Shirinskaya, G. Horowitz, J. Rivnay, G. G. Malliaras, and Y. Bonnassieux, Biosensors 8, 103 (2018).

${ }^{49}$ M. Z. Szymanski, D. Tu, and R. Forchheimer, IEEE Trans. Electron Devices 64 5114 (2017).

${ }^{50}$ V. Kaphle, P. R. Paudel, D. Dahal, R. K. Radha Krishnan, and B. Lüssem, Nat. Commun. 11, 2515 (2020).

${ }^{51}$ Y. Xu, H. Sun, and Y. Y. Noh, IEEE Trans. Electron Devices 64, 1932 (2017).

${ }^{52}$ H. Klauk, Adv. Electron. Mater. 4, 1700474 (2018).

${ }^{53}$ D. Braga, M. Ha, W. Xie, and C. D. Frisbie, Appl. Phys. Lett. 97, 193311 (2010).

${ }^{54}$ J. Lee, L. G. Kaake, H. J. Cho, X. Y. Zhu, T. P. Lodge, and C. D. Frisbie, J. Phys. Chem. C 113, 8972 (2009).

${ }^{55}$ V. Kaphle, S. Liu, A. Al-Shadeedi, C.-M. Keum, and B. Lüssem, Adv. Mater. 28, 8766 (2016).

${ }^{56}$ A. F. Paterson, H. Faber, A. Savva, G. Nikiforidis, M. Gedda, T. C. Hidalgo, X. Chen, I. McCulloch, T. D. Anthopoulos, and S. Inal, Adv. Mater. 31, 1902291 (2019).

${ }^{57}$ C. Cea, G. D. Spyropoulos, P. Jastrzebska-Perfect, J. J. Ferrero, J. N. Gelinas, and D. Khodagholy, Nat. Mater. 19, 679 (2020).

${ }^{58}$ A. G. Polyravas, V. F. Curto, N. Schaefer, A. B. Calia, A. Guimera-Brunet, J. A. Garrido, and G. G. Malliaras, Flexible Printed Electron. 4, 044003 (2019).

${ }^{59}$ R. B. Rashid, R. J. Ciechowski, and J. Rivnay, Flexible Printed Electron. 5, 014007 (2020). 